03,06

\title{
Фотогальванические токи и электрическая неоднородность 2-D-структурированного монокристалла сегнетоэлектрика-полупроводника
}

\section{(C) А.П. Одринский}

Институт технической акустики НАН Беларуси, Витебск, Беларусь

E-mail: a.odrinsky@gmail.com

Поступила в Редакцию 25 марта 2021 г.

В окончательной редакции 30 марта 2021 г.

Принята к публикации 30 марта 2021 г.

\begin{abstract}
Описаны условия эксперимента, позволившего прояснить механизм формирования электрической неоднородности кристалла $\mathrm{TlGaSe}_{2}$. Исследована эволюция аномальной реакции кристалла на фотовозбуждение и предложена интерпретация основных процессов определяющих динамику сигнала. Показано, что доминирующий вклад в развитие аномалий и формирование электрической неоднородности кристалла вносит фотогальваническая эдс. Также рассмотрено участие глубокоуровневых дефектов, как центров локализации заряда, в формировании электрической неоднородности слоистого кристалла сегнетоэлектрикаполупроводника.
\end{abstract}

Ключевые слова: фотогальванический ток, слоистые кристаллы, сегнетоэлектрики-полупроводники, центры локализации заряда.

DOI: 10.21883/FTT.2021.08.51148.062

\section{1. Введение}

Расширение сферы применения сегнетоэлектриков в твердотельной электронике [1] стимулирует углубление понимания явлений, связанных с пространственными неоднородностями локализации и делокализации заряда в этих материалах, а также наработку соответствующих методик изучения их электронной подсистемы. При исследовании электрической активности центров локализации заряда (ЦЛЗ) в сегнетоэлектрическом кристалле методы нестационарной спектроскопии (DLTS), развитые в рамках полупроводникового материаловедения, сталкиваются с проблемой неконтролируемой активности внутренних электрических полей, обусловленных доменной структурой. Традиционные исследования ЦЛЗ в сегнетоэлектриках методом термостимулированных токов также подвержены модификации сигнала под влиянием доменной структуры [2]. В то же время неравновесное заполнение ЦЛЗ при возбуждении кристалла светом дает уникальную возможность изучения активности ЦЛЗ в условиях малого возмущения доменной структуры [3]. Впервые проведенные систематические исследования на основе фотоэлектрической релаксационной спектроскопии (PICTS [4]) процессов тепловой делокализации зарядов с ЦЛ3 в 2D-структурированных монокристаллах сегнетоэлектриков-полупроводников $\mathrm{TlGaSe}_{2}$ и $\mathrm{TlInS}_{2}$ показали возможности и перспективность метода при изучении ЦЛ3 в этих кристаллах [2,3,5-10]. Был обнаружен набор идентичных процессов термоэмиссии. Вместе с тем наличие у кристалла сегнетоэлектрических свойств ведет к ощутимому увеличению вариации оценки эффективного сечения захвата ЦЛЗ и энергии активации делокализации [9]. Эти совместно определяемые оценки были строго коррелированны в соответствии с правилом Майер-Нельделя [11] и подвержены влиянию электрической неоднородности кристалла [3,9]. Участие обнаруженных центров в формировании пространственной неоднородности локализации заряда в кристалле подтвердилось идентичностью участков температуры регистрации термоэмиссии с ЦЛЗ и температуры, где наложение поляризующего поля существенно меняет ток деполяризации $\mathrm{TlGaSe}_{2}$ [7]. Заметим, что в области температур параэлектрической фазы $\mathrm{TlGaSe}_{2}$ $(T>120 \mathrm{~K})$ поляризованное состояние $\mathrm{TlGaSe}_{2}$ можно представить исключительно как пространственную вариацию заполнения ЦЛЗ, и это ставит вопрос о механизме формирования электрической неоднородности кристалла. Исследованиями электрически неоднородного $\mathrm{TlGaSe}_{2}$, поляризованного в низкотемпературной сегнетофазе [12], в релаксации фототока обнаружен процесс пространственного перераспределения носителей заряда в результате кулоновского взаимодействия. Это сопоставимо с пространственной вариацией заполнения ЦЛЗ. Однако параллельно наблюдались также аномалии фотоотклика подобные описанным ранее [13], вызванным фотогальваническим током (ФГТ). Но ФГТ также связан с нарушением электронейтральности кристалла [14] и, как следствие, сопровождается пространственным перераспределением свободных носителей заряда в процессе релаксации возбужденного состояния кристалла. Для выяснения механизма формирования электрической неоднородности возбуждаемого светом кристалла прове- 
дены исследования зависимости динамики фотоотклика $\mathrm{TlGaSe}_{2}$ от напряженности внешнего поля, а также энергии фотонов оптического возбуждения.

\section{2. Условия эксперимента}

Условия измерений и образец не отличались от описанных ранее $[6,7,9]$, исключая начальную подготовку эксперимента. Предварительно кристалл приводился в состояние близкое к термодинамическому равновесию при выдержке в темноте в течение часа при температуре $350 \mathrm{~K}$. Далее кристалл охлаждался без освещения до температуры $\sim 250 \mathrm{~K}$, соответствующей оптимуму наблюдения аномалий фотоотклика [13], и проводились измерения. Таким образом, эксперимент проходил в области температуры параэлектрической фазы $\mathrm{TlGaSe}_{2}$, что исключало возможность формирования электрической неоднородности образца в результате активности доменной структуры кристалла в низкотемпературной сегнетофазе. Кристалл имел $p$-тип проводимости с концентрацией носителей заряда $\sim 3 \cdot 10^{13} \mathrm{~cm}^{-3}$. Размеры образца составляли $2 \times 4 \times 0.3 \mathrm{~mm}$. Омические контакты формировались пайкой индием на торцевых поверхностях образца с выходом на фронтальную поверхность - плоскость скола. Расстояние между электродами составляло $3 \mathrm{~mm}$. Квазимонохроматическое излучение с энергией фотонов: $h v_{0} \sim 1.9 \mathrm{eV}\left(\Delta h v_{0} \sim 0.17 \mathrm{eV}\right) ; h v_{0} \sim 2.0 \mathrm{eV}$ $\left(\Delta h v_{0} \sim 0.21 \mathrm{eV}\right) ; \quad h v_{0} \sim 2.2 \mathrm{eV} \quad\left(\Delta h v_{0} \sim 0.26 \mathrm{eV}\right)$ подавалось перпендикулярно фронтальной поверхности образца. Плотность потока фотонов составляла $\sim 10^{15} \mathrm{~cm}^{-2} \mathrm{~s}^{-1}$. Использовалась типовая для регистрации фототока схема первичной измерительной цепи (ПИЦ) с подачей внешнего напряжения на последовательно соединенные образец и сопротивление нагрузки $1-33 \mathrm{~K} \Omega$.

Сбор данных о динамике фотоотклика проводился на основе специально развитого программного модуля интерактивного управления регистрацией данных путем последовательной регистрации кинетики фотоотклика через фиксированный интервал времени $\tau_{0}=10 \mathrm{~s}$. При регистрации проводилось поточечное накопление и усреднение сигнала реакции на возбуждение светом (2000 отсчетов, расположенных через фиксированный интервал времени $\Delta t=5 \cdot 10^{-5} \mathrm{~s}$ ) по 64 реализациям временной зависимости. Длительность отдельного процесса регистрации не превышала $\tau_{0}$, позволяя отслеживать эволюцию реакции кристалла на возбуждение светом, а также изменять $h v_{0}$ фотовозбуждения либо параметры ПИЦ на холостом периоде, в промежутках отсутствия регистрации данных.

\section{3. Результаты эксперимента}

На рис. 1 показана эволюция кинетики фотоотклика на возбуждение фотонами с $h v_{0}=1.9 \mathrm{eV}<E_{g}$ при ступенчатом переключении напряжения $U \sim 3-9 \mathrm{~V}$. Кривая 2 , полученная сразу после увеличения напряжения, фактически совпадает с кривой 1 , соответствующей установившемуся режиму периодического фотовозбуждения при $U \sim 3 \mathrm{~V}$. Для кривых 3 и 4 наблюдается постепенное увеличение фототока, сменяющееся выходом в установившийся режим, о чем свидетельствует фактическое совмещение кривых 4 и 5 с небольшим различием в релаксации сигнала. Приведенные на рис. 1 образцы кинетики фотоотклика хорошо сопоставимы с реакцией на фотовозбуждение, определяющейся изменением проводимости кристалла. При дальнейшем увеличении напряжения некоторые аномалии наблюдаются в кинетике релаксации, заметные для кривых 3 и 4 на рис. 2 и явно различимые на кривой 5 .

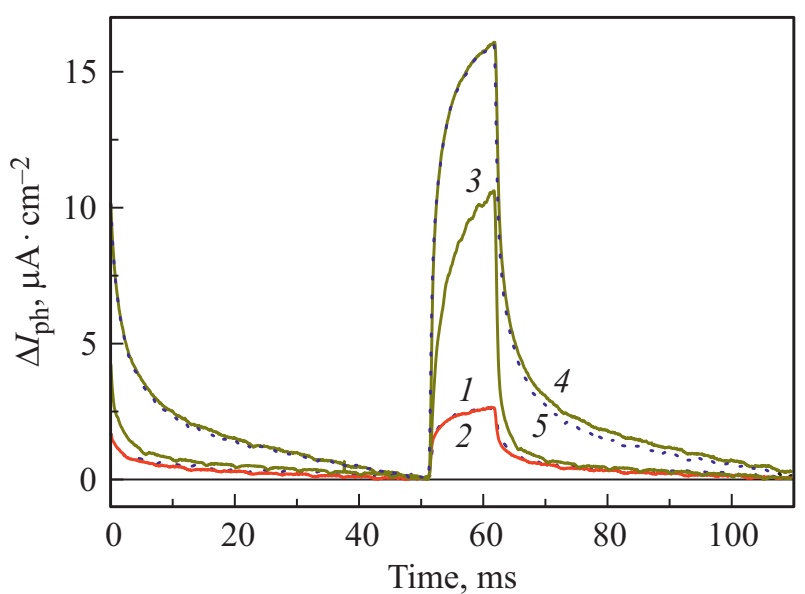

Рис. 1. Реакция кристалла на фотовозбуждение $(h v \sim 1.9 \mathrm{eV})$ при ступенчатом переключении приложенного напряжения с $U=3 \mathrm{~V}$ (кривая 1 ) до $9 \mathrm{~V}$ (кривые $2-5$ ). Кривая 2 регистрировалась непосредственно после переключения, кривые 3-5 по прошествии времени 10,20 и $30 \mathrm{~s}$ соответственно.

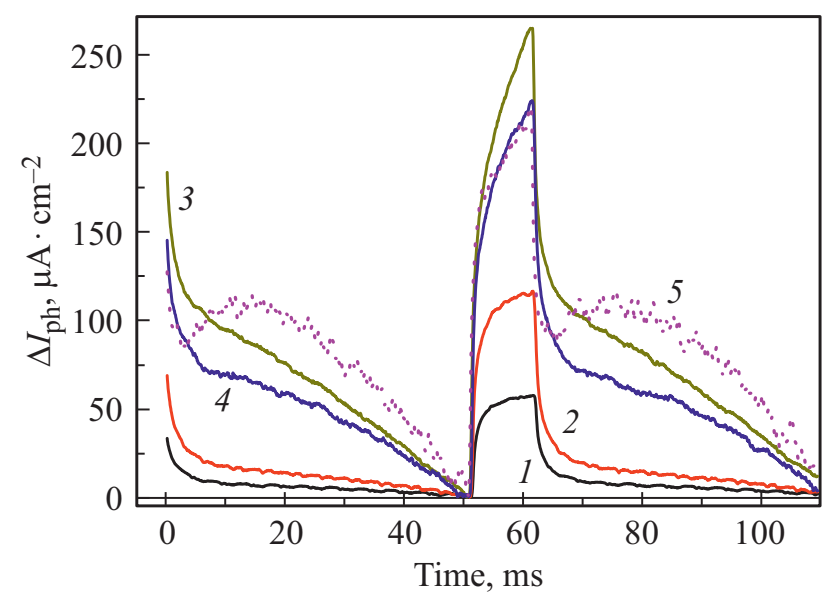

Рис. 2. Реакция кристалла на фотовозбуждение $(h v \sim 1.9 \mathrm{eV})$ при ступенчатом переключении приложенного напряжения с $U=21 \mathrm{~V}$ (кривая 1) до $48 \mathrm{~V}$ (кривые 2-4). Кривая 2 регистрировалась непосредственно после переключения, кривые 3 и 4 по прошествии времени 10 и $20 \mathrm{~s}$ соответственно. Кривая 5 регистрировалась по прошествии времени $10 \mathrm{~s}$ после переключения 56-66 V. 
Существенно меняется реакция кристалла на возбуждение светом на краю области собственного поглощения с энергией фотона $h v_{0}=2.0 \mathrm{eV} \sim E_{g}$ [15]. Если кривая 1 на рис. 3 , соответствующая установившемуся режиму периодического фотовозбуждения при $U \sim 3 \mathrm{~V}$, остается сопоставимой с изменением проводимости кристалла, то для кривых 2-5, полученных после ступенчатого переключения напряжения $U \sim 3-9 \mathrm{~V}$, наблюдается развитие аномалий фотоотклика. Отметим существенную трансформацию кинетики на начальном этапе $\sim 30 \mathrm{~s}$, сменяющуюся более вялыми изменениями (см. кривые 4 и 5). Последующее увеличение приложенного напряжения ведет к дальнейшему развитию аномалий фотоотклика кристалла, что иллюстрирует рис. 4. Здесь заметно развитие аномалий и отсутствие тенденции выхода в установившийся режим колебаний.

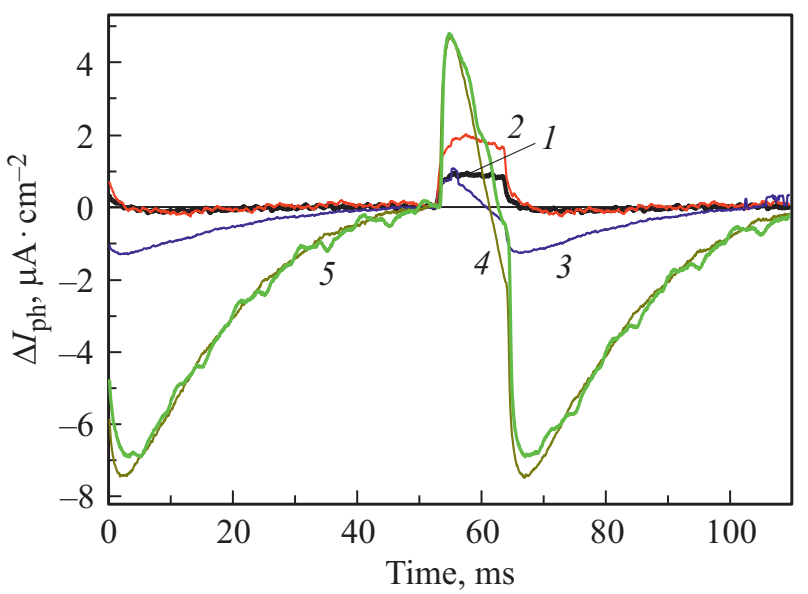

Рис. 3. Реакция кристалла на фотовозбуждение $(h v \sim 2.0 \mathrm{eV})$ при ступенчатом переключении приложенного напряжения c $U=3 \mathrm{~V}$ (кривая 1 ) до $9 \mathrm{~V}$ (кривые 2-5). Кривая 2 регистрировалась непосредственно после переключения, кривые 3-5 по прошествии времени 10,20 и $100 \mathrm{~s}$ соответственно.

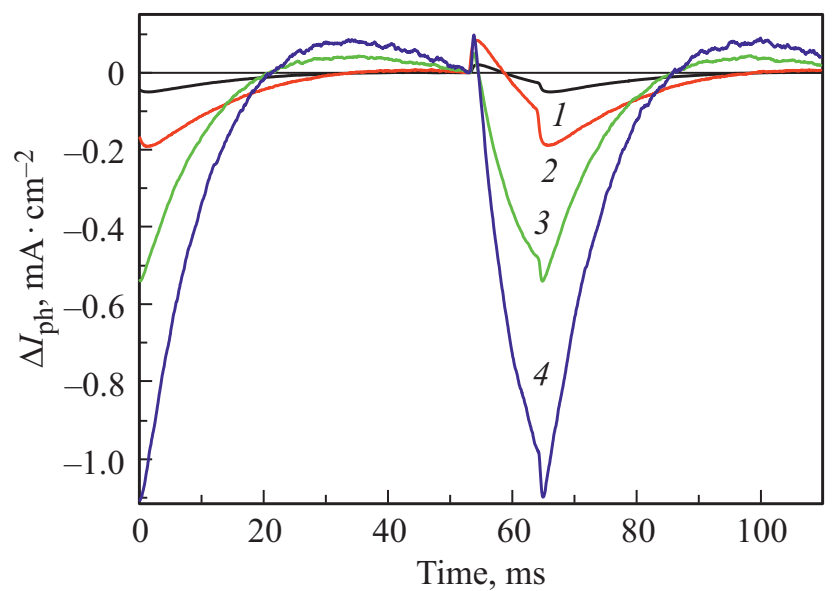

Рис. 4. Реакция кристалла на фотовозбуждение $(h v \sim 2.0 \mathrm{eV})$ при ступенчатом переключении приложенного напряжения с $U=21 \mathrm{~V}$ (кривая 1 ) до $48 \mathrm{~V}$ (кривые $2-4$ ). Кривая 2 регистрировалась непосредственно после переключения, кривые 3 и 4 по прошествии времени 40 и $50 \mathrm{~s}$ соответственно.

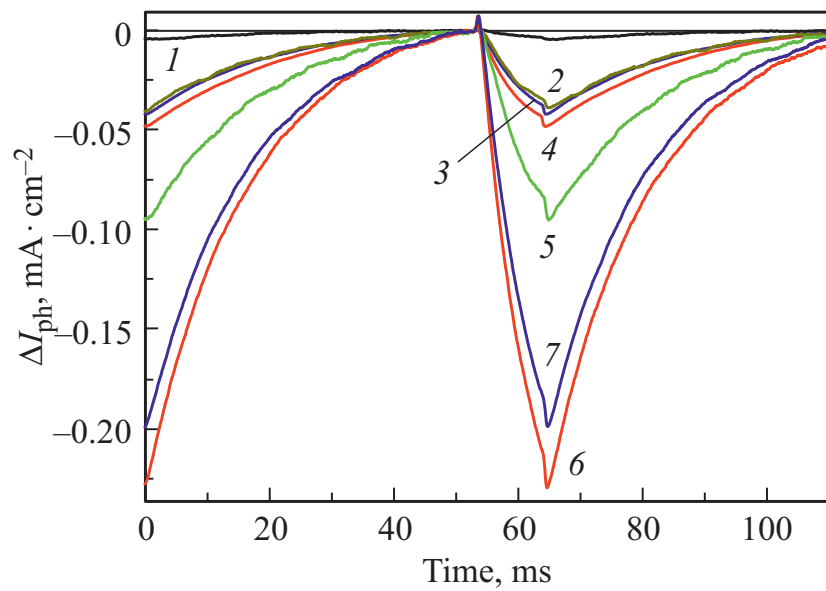

Рис. 5. Реакция кристалла на фотовозбуждение $(h v \sim 2.2 \mathrm{eV})$ при ступенчатом переключении приложенного напряжения с $U=9 \mathrm{~V}$ (кривые 1-4) до $21 \mathrm{~V}$ (кривые 5-7). Кривая 1 регистрировалась непосредственно после переключения 3-9 V, остальные кривые нумерованы в соответствии с последовательностью их регистрации через интервал $\tau_{0}=10 \mathrm{~s}$.

На рис. 5 показана эволюция реакции на возбуждение c $h v_{0} \sim 2.2 \mathrm{eV} \geq E_{g}$ при переключении $U \sim 3-9-21 \mathrm{~V}$. В отличие от рис. 4 здесь наблюдается относительно быстрая стабилизация режима изменений сигнала. При дальнейшем увеличении энергии фотонов вплоть до $h v_{0} \sim 2.5 \mathrm{eV}$ характер реакции на освещение, а также ее поведение при переключении внешнего напряжения существенно не изменяются. Таким образом, на кристалле $\mathrm{TlGaSe}_{2}$, приведенном в состояние близкое к термодинамическому равновесию, в окрестности температуры $T \sim 250 \mathrm{~K}$, при возбуждении светом с энергией фотонов $h v_{0} \geq E_{g}$ наблюдается развитие реакции, аномальной для полупроводникового кристалла. Освещение кристалла ведет к изменению знака фототока, явлению, известному как „отрицательная фотопроводимость“ ${ }^{\circ}[16]$. Развитие или эволюция реакции кристалла на фотовозбуждение достаточно медленная, с выходом в стабилизировавшийся режим изменений сигнала за время порядка десятков секунд и более. Это хорошо согласуется с сообщением о небольшой подвижности носителей заряда в $\mathrm{TlGaSe}_{2}$ [17], а также предполагаемым формированием областей избыточной концентрации свободных носителей [13], сопровождающимся нарушением локальной электронейтральности кристалла [12].

\section{4. Обсуждение результатов}

Обсуждение удобно начать с рис. 3, где реакция на свет, сопоставимая изменению проводимости при фотоинжекции свободных носителей, трансформируется в аномальную реакцию, развивающуюся с увеличением напряженности внешнего поля. В стабилизировавшемся режиме (кривые 4 и 5 ) в аномальном фотоотклике начальный рост сменяется устойчивым спадом сигнала, 
включая изменение знака. Формально эту реакцию следует сопоставить нарастанию тока в направлении противоположном току проводимости кристалла, заданному наложением внешнего поля. Для кривых 4 и 5 нарастание является фактически линейным процессом, протекающим со скоростью изменения тока $d I / d t \sim 10 \mu \mathrm{A} / \mathrm{s}$. Такой процесс может быть вызван исключительно действием сторонней эдс, возникающей при освещении кристалла. Более чем десятикратное увеличение амплитуды фотоотклика (ср. кривые 1 и 5) при лишь троекратном увеличении приложенного напряжения с 3 до $9 \mathrm{~V}$ также свидетельствует в пользу фотовольтаической природы аномалий. Отметим, что на всех аномальных зависимостях рисунка 3 остается заметным начальный рост сигнала, характерный и для нормальной реакции, т.е. фотоинжекции свободных носителей заряда, а в релаксации сигнала различим начальный участок спада, сопоставимый с рекомбинацией неравновесных носителей. Эти особенности начальных этапов нарастания и спада фотоотклика заметны и при дальнейшем увеличении прилагаемого напряжения, а также энергии фотонов (см. рис. 4 и 5). Можно полагать, что аномальная реакция некоторым образом включает составляющую, обусловленную изменением проводимости кристалла, что не характерно для фотовольтаической эдс, обусловленной полем локализованных зарядов (барьерной фото-эдс), но совместимо с присутствием фотогальванической эдс или внутреннего электрического поля, обусловленного избытком концентрации свободных носителей заряда. Доминирующий процесс нарастания обратно направленного тока наблюдается и при дальнейшем увеличении прилагаемого напряжения и энергии фотонов (рис. 4 и 5), что также согласуется с ФГТ как основной составляющей реакции кристалла на свет. Действительно, в отличие от фотовольтаического тока, обусловленного встроенным внутренним полем кристалла, ФГТ напрямую связан с пространственным перераспределением свободных носителей заряда под действием сил не электрической природы или сторонних сил [14], т. е. нарушением локальной электронейтральности кристалла и преодолением силы кулоновского взаимодействия. Но для формирования области избыточной концентрации свободных носителей до ощутимой величины необходимо определенное время, что хорошо сопоставимо с наблюдающимся процессом нарастания обратно направленного тока. Сопутствие кулоновского взаимодействия или ток экранирования можно обнаружить, рассматривая кинетику сигнала в темновой период релаксации возбуждения. На рис. 6 в логарифмических координатах представлены участки релаксации сигнала с рис. 3 и 5 . Исключая обсуждавшийся выше начальный этап релаксации, графики фактически соответствуют линейной зависимости как в стабилизировавшемся режиме изменений сигнала (кривые 2,3,4), так и в „плывущем“ нестабилизировавшемся режиме (кривые 1,5,6). Таким образом, в релаксации присутствует единственный процесс экспоненциального спада, сопоставимый исключительно с током экранирования, направленным на вос-

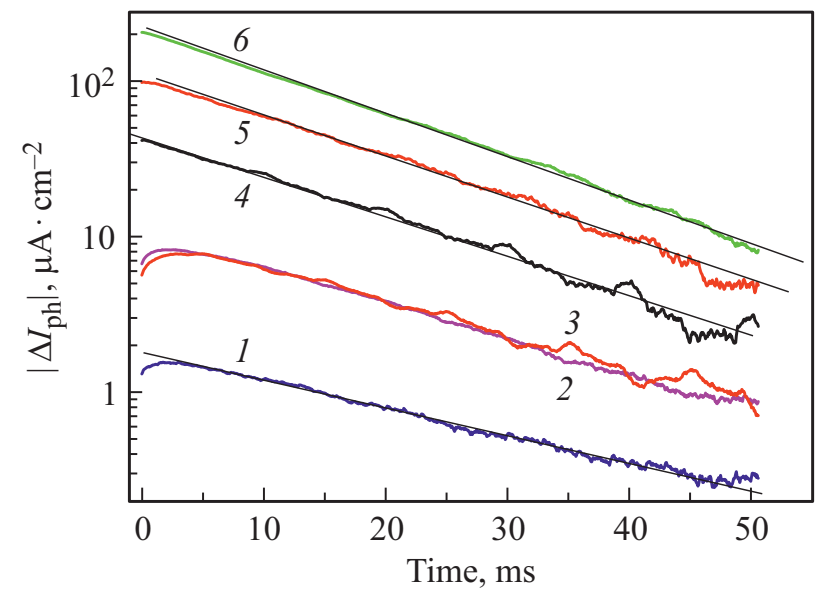

Рис. 6. Релаксация фотовозбуждения с $h v \sim 2.0 \mathrm{eV}$ (кривые $1-3$ ) и $h v \sim 2.2 \mathrm{eV}$ (кривые 4-6). Кривые $1-4$ регистрировались после переключения приложенного напряжения с $U=3 \mathrm{~V}$ до $9 \mathrm{~V}$ по прошествии времени $10,20,100$ и $30 \mathrm{~s}$, соответственно. Кривая 5 регистрировалась непосредственно после переключения приложенного напряжения с $U=9 \mathrm{~V}$ до $21.3 \mathrm{~V}$, а кривая 6 по прошествии времени $20 \mathrm{~s}$.

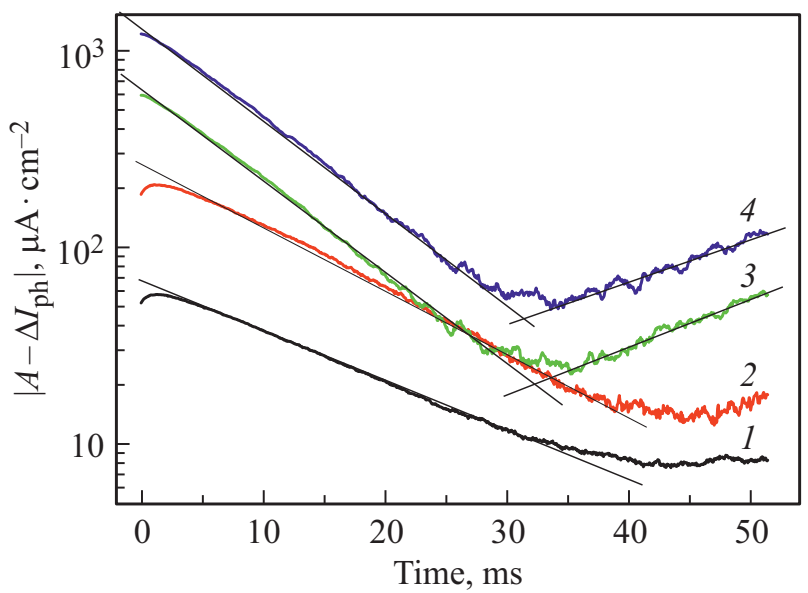

Рис. 7. Релаксация фотовозбуждения с $h v \sim 2.0 \mathrm{eV}$. Кривая 1 регистрировалась по прошествии времени $40 \mathrm{~s}$, после переключения приложенного напряжения с $U=9 \mathrm{~V}$ до $21.3 \mathrm{~V}$. Кривая 2 регистрировались непосредственно после переключения приложенного напряжения с $U=21.3 \mathrm{~V}$ до $48 \mathrm{~V}$, а кривые 3 и 4 по прошествии времени 40, $50 \mathrm{~s}$ соответственно.

становление электронейтральности кристалла. Доминирующим процессом реакции кристалла, развивающейся в условиях периодического возбуждения светом с $h v_{0}>2.0 \mathrm{eV}$, является действие фотогальванической эдс, определяющее динамику фотоотклика.

На рис. 7 показана релаксация сигнала в нестабилизировавшемся режиме, возникшем при увеличении напряженности внешнего поля и возбуждении $h v_{0} \sim 2.0 \mathrm{eV}$ (см. рис. 4). Здесь индивидуальный подбор параметра $A$ - постоянной смещения - выявил дополнительный вклад иного, менее интенсивного конкурирующего процесса релаксации, также обладающего экспоненци- 
альной зависимостью от времени. Конкуренция с более медленным процессом объясняет отсутствие выхода в стабилизировавшийся режим в условиях нашего эксперимента. Этот менее интенсивный процесс логично сопоставить вторичной реакции образца - релаксации неравновесного заполнения ЦЛЗ. Локализация заряда на ЦЛЗ в области формируемого ФГТ избытка или недостатка свободных носителей заряда способна внести свой вклад в кинетику тока экранирования. Но это справедливо для центров, обладающих интенсивным темпом делокализации зарядов при данной температуре. С другой стороны, центры с малым темпом делокализации зарядов такой способностью не обладают. В условиях периодического возбуждения такие центры будут накапливать заряд, формируя со временем пространственные вариации заполнения ЦЛЗ. В пользу предложенной интерпретации дополнительного процесса релаксации свидетельствует его переходной характер. Он не наблюдается в дальнейшем, в релаксации возбуждения при $h v_{0} \sim 2.2 \mathrm{eV}$, где сигнал снова представим моноэкспоненциальной зависимостью (см. кривые 4-6 на рис. 6). А, сравнив рис. 4 и 5, можно также заметить и соответствующее смещение уровня „нуля“, хорошо сопоставимое с формированием пространственных вариаций заполнения ЦЛЗ.

Отметим, что наблюдение аномалий фотоотклика на кристалле, приведенном в состояние близкое к термодинамическому равновесию и не охлаждавшемся до температуры сегнетофазы, вскрывает причинно-следственные связи. Этот факт свидетельствует о возникновении электрической неоднородности кристалла под действием фотогальванической эдс как первичном явлении, ведущем к нарушению локальной электронейтральности кристалла. Тогда как вариации заполнения ЦЛЗ - явление вторичное, вызванное определенной продолжительностью освещения кристалла и соответственно действием фотогальванической эдс, ведущей к накачке вариаций концентрации неравновесных свободных носителей заряда.

Корректность определения нулевого уровня сигнала важна при оценке величины, а также соотношения протекающих в кристалле токов. Для наших зависимостей „нулевой уровень“ выбран с определенной долей условности, по последней точке релаксации сигнала, регистрируемого в ПИЦ. Основанием такого выбора служили представления термодинамики о релаксации возбуждения и соответственно наибольшей близости состояния кристалла в указанный момент к равновесному. Однако это ведет к неучтенной остаточной погрешности. В рамках неопределенности, связанной с этой погрешностью, интегрируя ток за периоды светового возбуждения и релаксации, можно оценить соответствующий заряд, прошедший в ПИЦ, а также соотношение заряда, прошедшего за периоды релаксации и фотовозбуждения $-K$. Оказывается, что для зависимостей 4 и 5 на рис. 3 заряд, прошедший за время релаксации, приблизительно пятикратно превышает заряд, прошедший за время действия освещения ( $K=6.52$ и 4.59 соответственно). Аналогичная ситуация имеет место и для зависимостей на рис. $5 \mathrm{c}$ приблизительно троекратным превышением $(K=2.92$ и 3.02, для кривых 6 и 7 соответственно), а также данных рис. 4 ( $K=1.63$ и 1.64, для кривых 3 и 4 соответственно). Из этого следует, что в период возбуждения мы регистрируем только часть генерируемого сторонними силами тока, протекающего в кристалле, т.е. действие „эффективной“ эдс, а генерируемый при фотовозбуждении ток не коллинеарен току проводимости, задаваемому наложением внешнего поля. Выделив продольную и поперечную составляющие фотогальванического тока, можно заключить, что на начальной стадии наблюдения аномалий (рис. 3) поперечная составляющая ФГТ существенно (по крайней мере, на фактор четыре) превышает продольную составляющую. Учитывая квазидвумерный характер кристаллической структуры $\mathrm{TlGaSe}_{2}$, еe существенную анизотропию, можно полагать, что преимущественным направлением действия поперечной составляющей ФГТ является направление перпендикулярное слоям кристалла. В данном направлении проводимость на три порядка меньше проводимости по слою $[17,18]$, что создает выгодные условия как для формирования вариаций концентрации свободных носителей заряда, так и для ощутимости в эксперименте действия сторонних сил, не зависящих от проводимости кристалла [19].

Уменьшение $K$, или соотношения продольной и поперечной составляющих ФГТ при возбуждении с $h v_{0} \sim 2.2 \mathrm{eV}$ (рис. 5), хорошо согласуется с формированием вариаций заполнения ЦЛ3, как вторичного явления, компенсирующего действие ФГТ и обусловленного определенной продолжительностью удержания вариаций концентрации свободных носителей в направлении преимущественного действия ФГТ - перпендикулярно слоям кристалла.

Отметим, что межслоевые вариации концентрации неравновесных носителей в кристалле $\mathrm{TlGaSe}_{2}$ наблюдались и в индуцированном поглощении свободными носителями заряда [6], как появление модуляции бугеровского спада концентрации носителей вглубь кристалла после прерывания импульса оптической накачки, заметные уже за время $\sim 2 \mu \mathrm{s}$. Интерпретация этих данных как пространственной модуляции времени жизни неравновесных носителей, по сути, не отличается от предлагаемой - пространственной модуляции заполнения ЦЛЗ или модуляции потенциального рельефа кристалла. Исследования динамики краевой люминесценции монокристалла $\mathrm{TlInS}_{2}$ [20], изоструктурного $\mathrm{TlGaSe}_{2}$ также свидетельствуют о межслоевом разделении свободных электронов и дырок. Однако предложенная авторами [20] модель, основанная на образовании протяженных дефектов, в частности нарушений последовательности чередования слоев, не объясняет формирование электрической неоднородности кристалла. Действительно, само по себе образование дефекта или ЦЛЗ, изменение его зарядового состояния не ведет к нарушению локальной электронейтральности кристалла, для чего требуется действие сторонних сил, т. е. сил не электрической природы. Наши данные демонстрируют, что в условиях периодического 
возбуждения с $h v \geq E_{g}$ электрическая неоднородность $\mathrm{TlGaSe}_{2}$ формируется как результат действия ФГТ за относительно непродолжительное время, достаточное для формирования вариаций заполнения ЦЛЗ.

\section{5. Заключение}

Таким образом, в изучении эволюции фотоотклика кристалла $\mathrm{TlGaSe}_{2}$ с изменением напряженности прилагаемого внешнего поля предложена интерпретация основных процессов, определяющих аномалии реакции на периодическое фотовозбуждение с энергией фотонов $h v_{0} \geq E_{g}$. Показано, что в развитии аномальной реакции лежит задающее действие фотогальванической эдс, ведущее к нарушению электронейтральности кристалла и формированию пространственно-неоднородного заполнения ЦЛЗ кристалла.

При наложении внешнего поля вдоль слоев кристалла преимущественным направлением действия ФГТ является направление перпендикулярное слоям. Также ФГТ имеет компоненту, действующую в направлении противоположном току проводимости кристалла.

Для возникновения электрической неоднородности кристалла, сохраняющейся длительное время, достаточно формирования вариаций заполнения ЦЛЗ как вторичного явления, вызванного определенной продолжительностью освещения кристалла и соответственно действия фотогальванической эдс, ведущего к накачке вариаций концентрации неравновесных свободных носителей заряда.

Учитывая связанность фотогальванических токов или объемного фотовольтаического эффекта и фоторефракции $[21,22]$, представляет интерес перспектива управления двулучепреломлением $\mathrm{TlGaSe}_{2}$ путем варьирования подаваемого на кристалл внешнего напряжения.

Полученные результаты интересны также с точки зрения перспективы применения кристалла $\mathrm{TlGaSe}_{2}$, как фотовольтаического преобразователя энергии солнечного света. Принципиальным отличием нового типа преобразователя от известных предложений преобразователей на основе ФГТ в сегнетоэлектрических кристаллах $[23,24]$ является работа на поперечной составляющей фотогальванического тока. Предложенный преобразователь обладает преимуществом универсальности или самопроизвольной адаптация к номинальному напряжению подключаемой батареи аккумуляторов.

\section{Конфликт интересов}

Автор заявляет об отсутствии конфликта интересов.

\section{Список литературы}

[1] J.F. Scott. ISRN Mater. Sci. 2013, 187313 (2013).

[2] M.-H.Yu. Seyidov, А.П. Одринский, R.A. Suleymanov, Е. Acar, Т.Г. Мамедов, В.Б. Алиева. ФТТ 56, 10, 1964 (2014).
[3] А.П. Одринский, M.-H.Yu. Seyidov, R.A. Suleymanov, Т.Г. Мамедов, В.Б. Алиева. ФТТ 58, 4, 696 (2016).

[4] Ch. Hurter, M. Boilou, A. Mitonneau, D. Bois. Appl. Phys. Lett. 32, 821 (1978).

[5] M.Y. Seyidov, R.A. Suleymanov, F.A. Mikailzade, E. Kargın, A. Odrinsky. J. Appl. Phys. 117, 224104 (2015).

[6] V. Grivickas, A. Odrinski, V. Bikbajevas, K. Gulbinas. Phys. Status Solidi B 250, 1, 160 (2013).

[7] M.Y. Seyidov, F.A. Mikailzade, T. Uzun, A.P. Odrinsky, E. Yakar, V.B. Aliyeva, S.S. Babayev, T.G. Mammadov. Physica B: Condens. Matter, 483, 82 (2016).

[8] M.-H.Yu. Seyidov, R.A. Suleymanov, A.P. Odrinsky, A.I. Nadjafov, T.G. Mammadov, E.G. Samadli. JJAP. 50, 5, 05FC08-1 (2011).

[9] А.П. Одринский. ФТТ 56, 2, 331 (2014).

[10] А.П. Одринский, Т.Г. Мамедов, М.-Н.Yu Seyidov, В.Б. Алиева. ФТТ 56, 8, 1554 (2014).

[11] A. Yelon, B. Movaghar, R.S. Crandall. Rep. Prog. Phys. 69, 4, 1145 (2006).

[12] А.П. Одринский. ФТТ 62, 596, 4 (2020).

[13] А.П. Одринский, М.-Н.Yu Seyidov, Т.Г. Мамедов, В.Б. Алиева. ФТТ 59, 3, 447 (2017).

[14] K. Buse. Appl.Phys. B 64, 273 (1997).

[15] V. Grivickas, V. Bikbajevas, P. Grivickas. Phys. Status Solidi B 243, 5, R31-R33 (2006).

[16] Pai-Chun Wei, Surojit Chattopadhyay, Min-De Yang, ShihChang Tong, Ji-Lin Shen, Chien-Yao Lu, Han-Chang Shih, Li-Chyong Chen, Kuei-Hsien Chen. Phys. Rev. B 81, 045306 (2010).

[17] A.F. Qasrawi, N.M. Gasanly. Mater. Res. Bull. 39, 1353 (2004).

[18] С.Н. Мустафаева, В.А. Алиев, М.М. Асадов. ФТТ 40, 4, 612 (1998).

[19] H. Hatada, M. Nakamura, M. Sotome, Y. Kaneko, N. Ogawa, T. Morimoto, Y. Tokura, M. Kawasaki. PNAS, Proc. Natl. Acad. Sci. USA 117, 34, 20411-5 (2020).

[20] V. Grivickas, P. Scajev, V. Bikbajevas, O.V. Korolik, A.V. Mazanik. Phys. Chem. Chem. Phys. 21, 2102 (2019).

[21] A.M. Glass, D. Von der Linde. Ferroelectrics 10, 163 (1976).

[22] В.М. Фридкин, Б.П. Попов. УФН 126, 4, 657 (1978).

[23] L.Z. Tan, F. Zheng, S.M. Young, F. Wang, S. Liu, A.M. Rappe. Npj Comput. Mater. 2, 16026 (2016).

[24] K.T. Butler, J.M. Frost, A. Walsh. Energy Environ. Sci. 8, 838 (2015).

Редактор Ю.Э. Китаев 